\title{
Bacterial keratitis: Photodynamic inactivation reduced experimental inflammation
}

\author{
MONA DEICHELBOHRER ${ }^{1}$, MING-FENG WU ${ }^{2}$, BERTHOLD SEITZ ${ }^{2}$, DIRK HÜTTENBERGER ${ }^{3}$, \\ MATTHIAS W. LASCHKE ${ }^{4}$, HANS-JOCHEN FOTH ${ }^{5}$, BODO WONNENBERG ${ }^{1}$, STEFAN WAGENPFEIL ${ }^{6}$, \\ CAROLA MEIER $^{1}$, MARKUS BISCHOFF $^{7}$ and THOMAS TSCHERNIG ${ }^{1}$ \\ ${ }^{1}$ Institute of Anatomy and Cell Biology, Medical Faculty, Saarland University; ${ }^{2}$ Department of Ophthalmology, \\ Saarland University Medical Center/UKS, D-66424 Homburg/Saar; ${ }^{3}$ APO-CARE Pharma GmbH, D-33647 Bielefeld; \\ ${ }^{4}$ Institute of Clinical and Experimental Surgery, Saarland University, Medical Faculty, D-66424 Homburg/Saar; \\ ${ }^{5}$ Department of Physics, University of Kaiserslautern, D-67663 Kaiserslautern; \\ ${ }^{6}$ Institute for Medical Biometry, Epidemiology and Medical Informatics, Saarland University; \\ ${ }^{7}$ Institute of Medical Microbiology and Hygiene, Saarland University, Medical Faculty, D-66424 Homburg/Saar, Germany
}

Received December 16, 2016; Accepted March 17, 2017

DOI: $10.3892 / e t m .2017 .5109$

\begin{abstract}
The successful treatment of bacterial keratitis remains an unsolved clinical problem. The current study aimed to establish a murine keratitis model and to investigate the effect of chlorin e6 (Ce6) and photodynamic inactivation (PDI) on corneal inflammation. The cornea of anesthetized mice was scratched and covered with a bacterial suspension of Pseudomonas aeruginosa. A paste containing Ce6 was applied to the cornea with subsequent exposure to specified light. Two days later the animals were sacrificed, and the globes were processed for light microscopy. Evaluation parameters were the maximal corneal thickness and the severity of the hypopyon. The maximal corneal thickness of $290 \pm 16 \mu \mathrm{m}$ in the infected and untreated group was significantly reduced to $220 \pm 8 \mu \mathrm{m}$ in the infected and treated group $(\mathrm{P}<0.05)$. In addition, the hypopyon was less severe in the infected and treated group. In conclusion, the present study indicates that PDI using Ce6 may be a potential approach to treat patients suffering with severe bacterial keratitis.
\end{abstract}

\section{Introduction}

Bacterial keratitis is a common cause of blindness. It activates pattern recognition receptors in the cornea causing inflammation. Leukocytes, predominantly neutrophils, infiltrate the cornea and the anterior chamber of the eye $(1,2)$. The infection

Correspondence to: Professor Thomas Tschernig, Institute of Anatomy and Cell Biology, Medical Faculty, Saarland University, 61 Kirrberger Strasse, D-66424 Homburg/Saar, Germany

E-mail: thomas.tschernig@uks.eu

Key words: bacterial keratitis, Pseudomonas aeruginosa, photodynamic inactivation, chlorin e6 and inflammation often lead to corneal ulcers and consecutive blindness. The estimated prevalence varies from 6.3 to 710 per 100,000 (1). The high incidence is due not only to poor hygiene standards and a greater risk of infection in developing countries, but also to the widespread use of contact lenses with associated complications in industrial nations $(3,4)$. The two primary gram-positive and gram-negative bacterial strains responsible for causing bacterial keratitis are Staphylococcus aureus and Pseudomonas aeruginosa (1). The first-line treatment is primarily performed using fluoroquinolones (1). Due to the resistance of bacteria to antibiotics, the effectiveness of the treatment of bacterial keratitis is decreasing. These circumstances have made research into the development of novel therapy approaches essential. Photodynamic inactivation (PDI) was rarely used as a treatment option for ocular infections, although other photodynamic therapy procedures including corneal crosslinking with riboflavin and ultraviolet light (UV-crosslinking), are established as a treatment for specific corneal diseases such as keratoconus (5-10). UV-crosslinking is a photochemical crosslinking of fibers within the corneal tissue.

Chlorin e6 [Ce6; C34H36N4O6 in full: (17S, 18S)18-(2-carboxyethyl)-20-(carboxymethyl)-12-ethenyl-7-ethyl-3, 8,13,17-tetramethyl-17,18,22,23-tetrahydroporphyrin-2-carboxylic acid] and PDI have previously been investigated in vitro and appear to be promising in the treatment of microbial keratitis $(11,12)$. PDI functions on the principle that the accumulation of a specific photosensitizer in the relevant tissue induces the generation of reactive oxygen species (ROS) when exposed to light of a specific wavelength. These ROS may kill bacteria and be the initial step in healing bacterial keratitis (13-17). The histopathology of bacterial infected mice indicates focal cellular infiltrates within the corneal stroma. Concomitant, heavy cellular infiltrates are identified in the anterior chamber, which is also called the hypopyon. Typically, in the later stages corneal ulcers occur (18). Corneal ulcers are estimated to cause approximately two million new cases of monocular blindness every year (1). 
The purpose of the present study was to establish experimental infectious keratitis in mice using pseudomonades and to investigate the effect of treatment with $\mathrm{Ce} 6$ on corneal inflammation.

\section{Materials and methods}

Animals. In the present study, a total of 30 C57BL6N mice (Janvier Labs, Saint-Berthevin Cedex; weight, 29.1 \pm 3.8 g; 24 females:6 males) were used. Mice used were 8-11 months old as older mice are more susceptible to experimental infection (19). The housing conditions were as follows: temperature, $22^{\circ} \mathrm{C}$; humidity, $60 \%$; and a $12 \mathrm{~h}$ light/dark cycle. The animals had access to chow and water ad libitum. The animal experiments were approved by the Government of the Saarland (Landesamt für Soziales, Gesundheit und Verbraucherschutz). The Ethical Committee for Animal Experiments of the Saarland approved the present study. One eye of each animal was infected or treated, the other one was untouched. All experiments were performed in accordance with the Guide for the Care and Use of Laboratory Animals (20).

Induction of keratitis. Under anesthesia $(0.05 \mathrm{mg} / \mathrm{kg}$ fentanyl, Hameln Pharma Plus GmbH, Hameln, Germany; $5 \mathrm{mg} / \mathrm{kg}$ midazolam, Hameln Pharma Plus $\mathrm{GmbH}$; and $0.5 \mathrm{mg} / \mathrm{kg}$ medetomidine hydrochloride, Orion Corporation, Espoo, Finland), three scratches of $\sim 2 \mathrm{~mm}$ in length were applied to the center of the cornea using a 27 -gauge needle. Subsequently, $5 \mu 1$ inoculum was pipetted onto the cornea and was left on the eye for $20 \mathrm{~min}$. Analgesia was applied daily by intraperitoneal injection with $4 \mathrm{mg} / \mathrm{kg}$ carprofen (Zoetis Schweiz GmbH, Zuerich, Schweiz). The inoculum consisted of multi-resistant Pseudomonas aeruginosa, strain 54, which had been cultured on blood agar plates (trypticase soy agar II, $5 \%$ sheep blood; BD GmbH, Heidelberg, Germany) for $24 \mathrm{~h}$ at $37^{\circ} \mathrm{C}$, then suspended in $10 \mathrm{ml} 2 \% \mathrm{BD}$ Difco Luria Bertani broth (LB broth; BD GmbH) for an overnight culture at $37^{\circ} \mathrm{C}$. From the overnight culture, $100 \mu \mathrm{l}$ was diluted in $10 \mathrm{ml} 2 \%$ LB broth for another $3 \mathrm{~h}\left(37^{\circ} \mathrm{C}\right)$ of culturing and finally the suspension was adjusted to an optical density of 10 for inoculation using routine procedures (densitometer). Post-infection treatment was performed for one pilot animal at $12 \mathrm{~h}$ and for the rest at $24 \mathrm{~h}$ under anesthesia as described above.

Treatment. For PDI, the infected eyes were incubated with a photosensitizer gel containing $0.1 \% \mathrm{Ce} 6$ or only the gel base (hydroxypropyl methylcellulose) for the sham-treated group (both APOCARE Pharma GmbH, Bielefeld, Germany) for $30 \mathrm{~min}$. From the PDI group, one mouse was treated once for $15 \mathrm{~min}$ and one pilot mouse was treated twice, at 12 and 24 h, with a high Ce6 concentration (2.0\%) for 30 min. In six randomly selected animals the epithelium of the cornea was removed with a hockey knife prior to PDI. The procedure was completed in darkness to avoid premature activation of the photosensitizer. The ointment was removed using a cotton swab and the animals were subsequently exposed to red light of a wavelength of $670 \mathrm{~nm}$ for $6 \mathrm{~min}$.

Morphology. All animals were sacrificed by neck dissection whilst under deep anesthesia as described above
3 days' post infection and the globes were enucleated and histologically processed as follows: Fixation was performed using Davidson-Solution, (AppliChem GmbH, Darmstadt, Germany) for $6 \mathrm{~h}$. Subsequently, samples were left $12 \mathrm{~h}$ in isopropanol (30\%, in aqua dest distilled water) on a shaker. The samples were embedded in paraffin using routine procedures. Sections were performed and mounted on glass slides using routine procedures and the mounted sections were stained with hematoxylin and eosin. For evaluation, five sections of $5 \mu \mathrm{m}$ thickness were obtained from the center region (with a distance of $50 \mu \mathrm{m}$ from each other; Fig. 1A). The location of the maximum corneal thickness was selected and measured using cellSens Standard software (version 1.8.1; Olympus Deutschland GmbH, Düsseldorf, Germany). Fig. 1B indicates a representative measurement of the maximal corneal thickness. Mean values per group were calculated. In the data figures, each symbol represents one animal. For evaluation, a light microscope was used (magnification, x100; Axiophot, Zeiss, Jena, Germany).

Evaluation. The severity of hypopyon was evaluated in a blinded manner using a novel semi-quantitative scoring from $0-3$ as follows: 0 points, no hypopyon (no cellular infiltrate within the anterior eye chamber); 1 point, mild hypopyon (cellular infiltrate partly filling the anterior eye chamber) 2 points, moderate hypopyon (cellular infiltrate filling the anterior eye chamber); and 3 points; severe hypopyon (heavy cellular infiltrate filling and distorting the anterior eye chamber), as presented in Fig. 2. In the preliminary study the final duration of the treatment was set at $30 \mathrm{~min}$. The pilot mice were included within the analyses of the experimental groups as no marked differences to mice within the same experimental group were observed. In summary, the groups for analyses were as follows: Uninfected/untreated $(n=6)$, infected/untreated $(n=4)$, infected/sham-treated $(n=8)$ and infected/PDI-treated $(n=12)$. Statistical analyses were performed using Prism 5 software (GraphPad Software Inc., La Jolla, CA, USA). Mann-Whitney U test for two independent samples was used for median comparison. Data are presented as mean \pm standard error of the mean and the two-sided level of significance was defined as $\mathrm{P}<0.05$.

\section{Results}

Corneal inflammation. The present study identified focal cellular infiltrates and inflammatory oedema within the corneal stroma in the infected non-treated, but also-to a lesser extent-in the infected-treated animals. No corneal ulcerations were observed within any of the experimental groups. The maximal corneal thickness of normal eyes of C57BL6N mice (uninfected/untreated) was $110 \pm 7 \mu \mathrm{m}$. The maximal corneal thickness of eyes from the infected/PDI-treated group was $220 \pm 8 \mu \mathrm{m}$, whereas $257 \pm 19 \mu \mathrm{m}$ was indicated for eyes of the infected/sham-treated group and $290 \pm 16 \mu \mathrm{m}$ for eyes of the infected/untreated group, all of which were significantly increased compared with the uninfected/untreated group (all $\mathrm{P}<0.05$ ). In addition, the maximal corneal thickness of the infected/untreated group was significantly increased compared with the infected/PDI-treated group $(\mathrm{P}<0.05$; Fig. 3). 

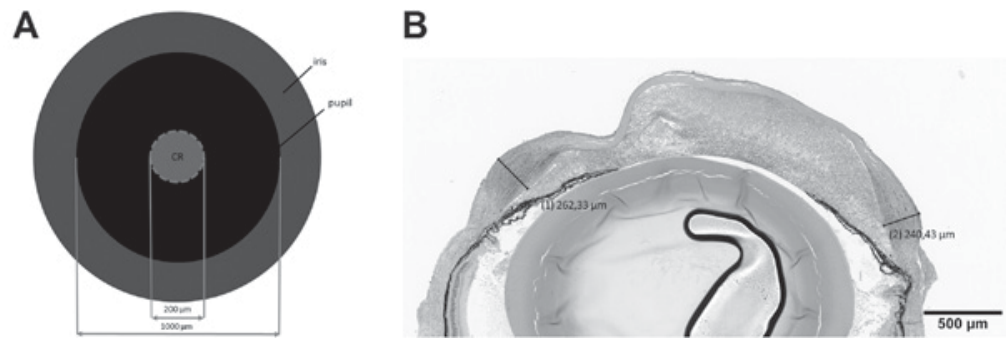

Figure 1. Schematic of obtaining histological sections. Magnification was indicated by the scale bar=1 $\mathrm{mm}$ per $500 \mu \mathrm{m}$. The diameter of the pupil was $\sim 1,000 \mu \mathrm{m}$. (A) The sections were obtained from the CR which had a diameter of $\sim 200 \mu \mathrm{m}$. (B) Representative microphotograph following hematoxylin and eosin staining with two scale bars to determine the maximal thickness of the cornea. CR, center region.
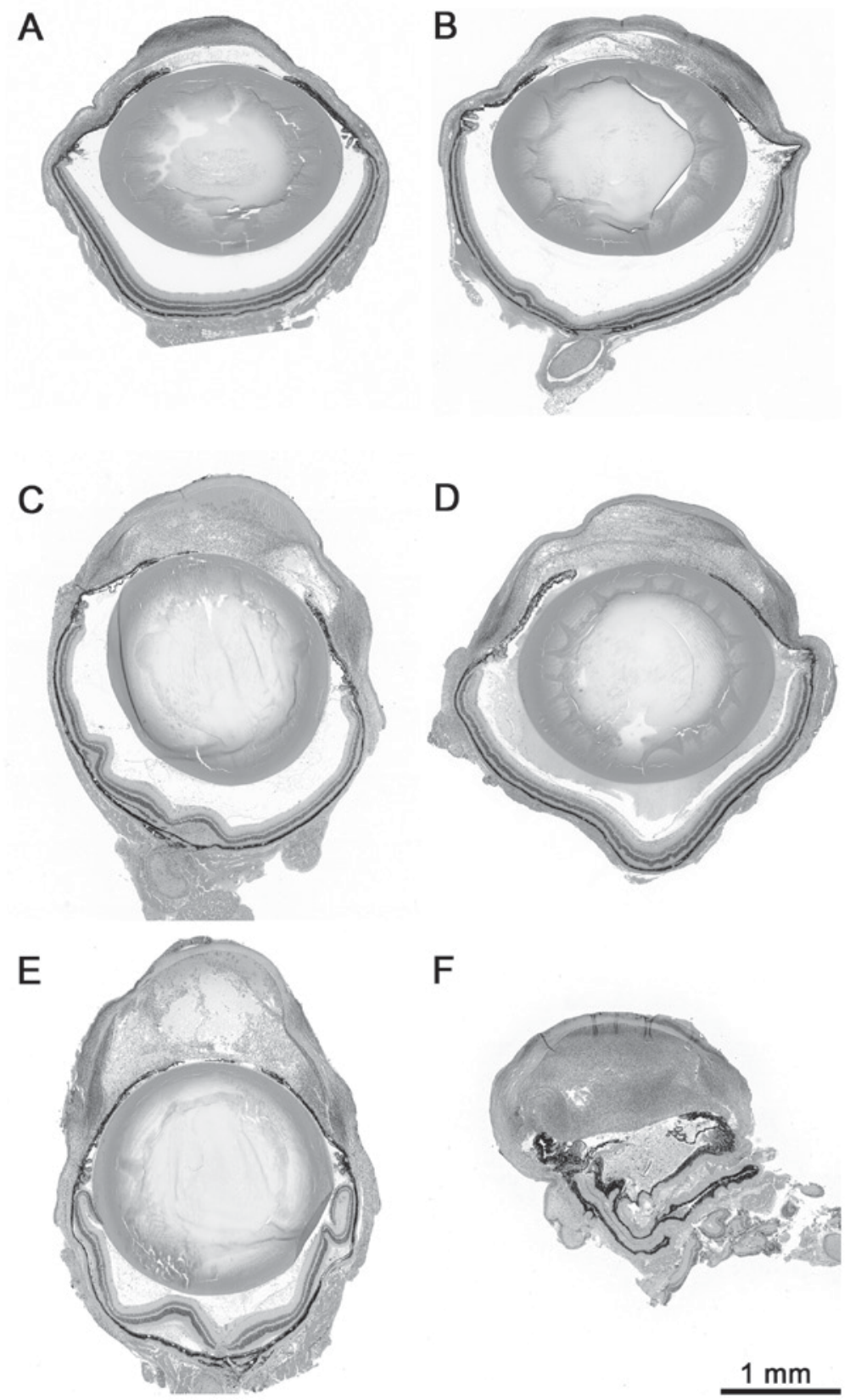

Figure 2. Typical examples for the severity of hypopyon from mild to moderate to severe. The score, indicating the severity of each hypopyon ranged from (A and B) 1, being mild, (C and D); 2, being moderate and (E and F) 3, being severe. Samples were stained with hematoxylin and eosin.

Hypopyon. Concomitant infiltrates of neutrophils (hypopyon/hypopya) were identified in the anterior chamber of the infected mice. The severity of hypopyon was significantly higher in the eyes of the infected/untreated group compared 

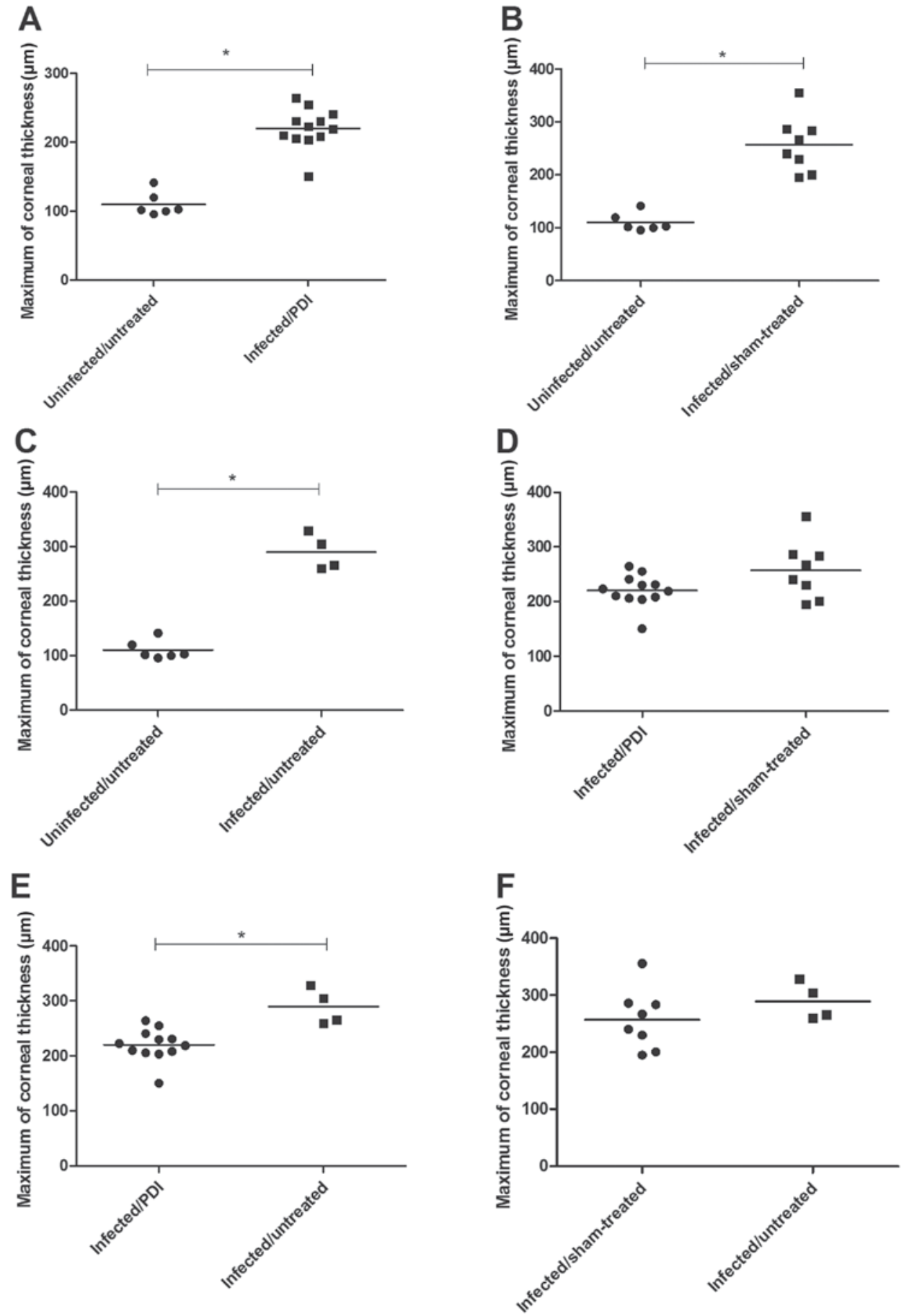

Figure 3. Comparison of the groups from the PDI experiments as indicated with regard to maximum corneal thickness. The maximal corneal thickness of the uninfected/untreated group compared with the (A) infected/PDI-treated, (B) infected/sham-treated group and (C) the infected/untreated group. The maximal corneal thickness of the infected/untreated group compared with the (D) infected/sham-treated, (E) infected/PDI-treated and (F) infected/sham-treated groups. ${ }^{*} \mathrm{P}<0.05$. Black bars represent the mean values and every point or square represents the mean value of one animal. PDI, photodynamic inactivation.

with those of the infected/PDI-treated group $(\mathrm{P}<0.05)$. Furthermore, hypopyon in the infected/sham-treated group was significantly less severe than in infected/untreated mice (Fig. 4).

\section{Discussion}

The determination of the dimension of the induced keratitis was difficult as a result of the inhomogeneous infiltration and inflammation. Due to the focal infiltrates and edema, a general increase in the thickness of the cornea was observed, but particularly at the sites of the focal infiltrates. As other methods, including counting the infiltrating cells or the determination of the whole corneal area on the sections failed, the authors of the current study selected the maximal corneal thickness as a measure for the extent of inflammation, following an evaluation of $>1,000$ sections, which indicated that the maximal thickness of the cornea represents the dimension of the inflammation. This indicates a drawback of the presented histological approach: The production of numerous 


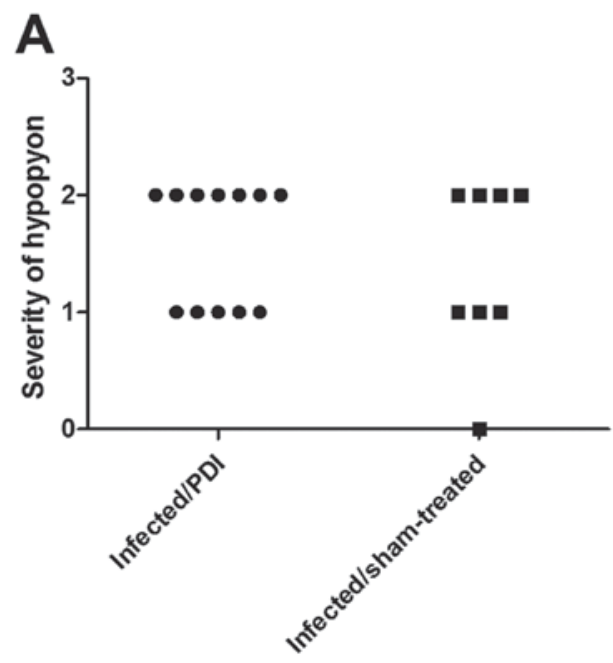

the most accurate measurement of inflammation in the view of the authors. The determination of colony forming units (CFU) would be an alternative and quantitative method but may also be unsatisfactory, as the number of CFU alone does not define keratitis. In addition, it would not be possible to isolate the cornea of the eyeball in the mouse, due to its small size. Most likely the eyeball would be homogenized and all compartments of the eye would be mixed together and the specific situation of the cornea would be uncertain. In the present study, the maximal corneal thickness in mice with keratitis was $20 \%$ less following PDI when compared with untreated mice. This indicated a beneficial effect of treatment using PDI that may be beneficial for the treated mice. However, the maximum corneal thickness following PDI therapy remained almost double that of uninfected animals. The disease course following treatment with PDI was not investigated in the present study. One PDI treatment may lead to healing or quicker healing of the keratitis. It is likely that a repetitive treatment using PDI may be necessary, and this should be determined in future experiments. Notably, the infected and sham-treated mice did not differ significantly from the animals undergoing treatment using PDI. This is difficult to understand; however, a possible explanation is that the sham treatment included a light exposure without the active drug. This exposure itself may inhibit the bacterial growth within the cornea. Another explanation would be that the paste (solvent) without the drug had antibacterial effects. However, the comparison between the sham-treated animals and untreated animals did not reveal a significant difference.

The hypopyon that occurred following the experimental infection varied in shape and extent. The score used for the evaluation was semi-quantitative. A weakness of this score is that there is not a well-defined distinction between score 1 and 2 or between score 2 and 3. Notably, PDI-treated infected eyes and sham-treated infected eyes had a less severe hypopyon score when compared with the infected but untreated eyes. This supported the hypothesis that the paste or the red light possessed anti-inflammatory or antibacterial effects. This may have accounted for an improvement of 1 point using the scoring system. However, the present results of the hypopyon indicated a reduction of hypopya after the use of therapy. It remains unclear, how the PDI influenced the hypopyon; however, the hypothesis is that $\mathrm{Ce} 6$ and the phototherapy reached the cornea and the anterior chamber, leading to inactivation of pseudomonades in both compartments. By contrast, the application of red light alone may have mediated the effect since less severe hypopyon was observed following sham treatment. Taken together, the present study presents preliminary data that using $\mathrm{Ce} 6$ and PDI may be an important treatment approach of severe bacterial keratitis.

Previously, it has been demonstrated in vitro that Ce6 and

Figure 4. Comparison of the groups from the PDI experiments as indicated with regard to severity of the hypopyon. Comparison of (A) the infected/PDI-treated and the infected/sham-treated groups, (B) the infected/PDI-treated group and the infected/untreated group and (C) the infected/sham-treated and infected/untreated groups with regard to the severity of hypopyon. ${ }^{*} \mathrm{P}<0.05$. Every point or square represents the mean value of one animal. PDI, photodynamic inactivation.

sections and staining and even the number of $\mathrm{h}$ spent investigating and measuring the slides is a limiting factor in those study designs. The maximal corneal thickness or diameter is keratitis depends on species and age of the experimental animals in addition to the bacteria used (22). In principal, it is challenging to use staphylococci in the murine keratitis model due to the difficulties in establishing infection. Even when using pseudomonades, the susceptibility varies in different age groups. In general, older mice are more susceptible than postnatal or young mice. Therefore, primarily older mice were selected for the present study. 
Effects of Ce6 treatment and PDI with regard to the appropriate time point and duration of incubation time should be repeated with higher numbers of animals. The present study was, to the best of our knowledge, the first to assess the feasibility and usefulness of PDI-therapy. Beyond the inflammation of the corneal tissue, the burden of living bacterial cells would be a noteworthy parameter. At the time of the present experiment, measurement of the bacterial burden of infected cornea was not completed. As mentioned, it is difficult to determine CFU within the isolated cornea. In a previous study on the use of a high mobility group box-1 inhibitor in a murine model of keratitis, the determination of CFU in isolated cornea was established (22). However, the CFU numbers determined in homogenates of whole eyeballs would also provide valuable information.

The results presented in the current study may serve as a promising basis for further investigations. The potential of this approach should be investigated with the aim of further development and optimization in ongoing studies in order to achieve the integration of this therapy in future standard clinical practice. In conclusion, $\mathrm{Ce} 6$ and PDI may be a potential approach for treatment of patients suffering from severe bacterial or acanthamoebiasis-keratitis, which is resistant against conventional therapy.

\section{Acknowledgements}

The authors of the present study would like to thank Mrs. Ann Soether for language editing and Mrs. Tina Wiesen-Philipps for help with the manuscript.

\section{References}

1. Ong HS and Corbett MC: Corneal infections in the 21st century. Postgrad Med J 91: 565-571, 2015.

2. Taube MA, del Mar Cendra M, Elsahn A, Christodoulides M and Hossain P: Pattern recognition receptors in microbial keratitis. Eye (Lond) 29: 1399-1415, 2015.

3. Young G, Young AG and Lakkis C: Review of complications associated with contact lenses from unregulated sources of supply. Eye Contact Lens 40: 58-64, 2014.

4. Lorenzo-Morales J, Khan NA and Walochnik J: An update on Acanthamoeba keratitis: Diagnosis, pathogenesis and treatment. Parasite 22: 10, 2015.

5. Makdoumi K, Mortensen J, Sorkhabi O, Malmvall BE and Crafoord S: UVA-riboflavin photochemical therapy of bacterial keratitis: A pilot study. Graefes Arch Clin Exp Ophthalmol 250: 95-102, 2012.

6. Stachon T, Wang J, Eppig T, Langenbucher A, Bischoff M, Seitz B and Szentmáry N: KGF, FGFb, VEGF, HGF and TGF $\beta 1$ secretion of human keratocytes following photodynamic inactivation (PDI) in vitro. Graefes Arch Clin Exp Ophthalmol 251: 1987-1993, 2013.
7. Stachon T, Wang J, Song X, Langenbucher A, Seitz B and Szentmáry N, Impact of crosslinking/riboflavin-UVA-photodynamic inactivation on viability, apoptosis and activation of human keratocytes in vitro. J Biomed Res 29: 321-325, 2015.

8. Wu MF, Stachon T, Wang J, Song X, Colanesi S, Seitz B Wagenpfeil S, Langenbucher A and Szentmáry N: Effect of keratocyte supernatant on epithelial cell migration and proliferation after corneal crosslinking (CXL). Curr Eye Res 41: 466-473, 2016.

9. Randleman JB, Khandelwal SS and Hafezi F: Corneal cross-linking. Surv Ophthalmol 60: 509-523, 2015.

10. Papaioannou L, Miligkos M and Papathanassiou M: Corneal collagen cross-linking for infectious keratitis: A systematic review and meta-analysis. Cornea 35: 62-71, 2016.

11. Wang J, Stachon T, Eppig T, Langenbucher A, Seitz B and Szentmáry N: Impact of photodynamic inactivation (PDI) using the photosensitizer chlorin e6 on viability, apoptosis and proliferation of human keratocytes in vitro. Graefes Arch Clin Exp Ophthalmol 251: 2725-2731, 2013.

12. Wang J, Stachon T, Eppig T, Langenbucher A, Seitz B and Szentmáry N: Impact of photodynamic inactivation (PDI) using the photosensitizer chlorin e 6 on viability, apoptosis, and proliferation of human corneal endothelial cells. Graefes Arch Clin Exp Ophthalmol 251: 1199-1204, 2013.

13. Rovaldi CR, Pievsky A, Sole NA, Friden PM, Rothstein DM and Spacciapoli P: Photoactive porphyrin derivative with broad-spectrum activity against oral pathogens In vitro. Antimicrob Agents Chemother 44: 3364-3367, 2000

14. Gad F, Zahra T, Francis KP, Hasan T and Hamblin MR: Targeted photodynamic therapy of established soft-tissue infections in mice. Photochem Photobiol Sci 3: 451-458, 2004.

15. Park JH, Moon YH, Bang IS, Kim YC, Kim SA, Ahn SG and Yoon JH: Antimicrobial effect of photodynamic therapy using a highly pure chlorin e6. Lasers Med Sci 25: 705-710, 2010.

16. Martins SA, Combs JC, Noguera G, Camacho W, Wittmann P, Walther R, Cano M, Dick J and Behrens A: Antimicrobial efficacy of riboflavin/UVA combination in vitro for bacterial and fungal isolates: A potential new treatment for infectious keratitis. Invest Ophthalmol Vis Sci 49: 3402-3408, 2008.

17. Khan YA, Kashiwabuchi RT, Martins SA, Castro-Combs JM Kalyani S, Stanley P, Flikier D and Behrens A: Riboflavin and ultraviolet light a therapy as an adjuvant treatment for medically refractive Acanthamoeba keratitis: Report of 3 cases. Ophthalmology 118: 324-331, 2011.

18. Ekanayaka SA, McClellan SA, Barrett RP, Kharotia S and Hazlett LD: Glycyrrhizin reduces HMGB1 and bacterial load in Pseudomonas aeruginosa keratitis. Invest Ophthalmol Vis Sci 57: 5799-5809, 2016.

19. Girgis DO, Sloop GD, Reed JM and O'Callaghan RJ: Susceptibility of aged mice to Staphylococcus aureus keratitis. Curr Eye Res 29: 269-275, 2004.

20. National Research Council (US) Committee for the Update of the Guide for the Care and Use of Laboratory Animals. Guide for the Care and Use of Laboratory Animals. 8th edition. National Academies Press (US), Washington (DC), 2011.

21. Pinto JG, Pereira AH, de Oliveira MA, Kurachi C, Raniero LJ and Ferreira-Strixino J: Chlorin E6 phototoxicity in L. major and L. braziliensis promastigotes-in vitro study. Photodiagnosis Photodyn Ther 15: 19-24, 2016 (In Press).

22. Marquart ME: Animal models of bacterial keratitis. J Biomed Biotechnol 2011: 680642, 2011. 